\title{
Effectiveness of thiazides on serum and urinary calcium levels and bone mineral density in patients with osteoporosis: a systematic review and meta-analysis
}

This article was published in the following Dove Press journal:

Drug Design, Development and Therapy

\author{
Lei Cheng' \\ Keyan Zhang' \\ Zhenyong Zhang ${ }^{2}$ \\ 'Department of Thyroid Surgery, \\ The First Affiliated Hospital of China \\ Medical University, Shenyang, Liaoning, \\ China; ${ }^{2}$ The Second Department of \\ Clinical Oncology, Shengjing Hospital \\ of China Medical University, Shenyang, \\ Liaoning, China
}

Objective: Osteoporosis is the most common metabolic bone disease and a major public health problem worldwide. Thiazides are widely used as antihypertensive agents with good tolerability and efficacy. Furthermore, thiazides have long been regarded as candidates for the prevention of postmenopausal bone loss. However, there is insufficient evidence that thiazides have a sustained beneficial effect on preserving bone mass and preventing osteoporosis to date.

Materials and methods: We searched the PubMed, the Cochrane Library, and Embase in June 2018 for randomized controlled trials on the use of thiazides to treat osteoporosis. Continuous outcomes are presented as the standardized mean difference (SMD) and 95\% CI. Furthermore, $P$-values $<0.05$ were considered significant.

Results: Five trials with 756 patients were randomly assigned in the five trials included in this meta-analysis. Serum calcium level was higher in the thiazide group than in the control group (SMD $0.33,95 \%$ CI $[0.16,0.50]$ ), and urinary calcium level was significantly lower in the thiazide group (SMD $-0.35,95 \%$ CI $[-0.52,-0.17]$ ). There was no significant difference in bone mineral density between the two groups (SMD 0.19, 95\% CI [-0.16, 0.54]).

Conclusion: Thiazides might play a role in preserving bone mass and be effective in the prevention and treatment of osteoporosis. Future high-quality trials are needed to confirm our findings in the future.

Keywords: thiazides, osteoporosis, serum calcium, urinary calcium, bone mineral density

\section{Introduction}

Osteoporosis is a chronic systemic skeletal disorder characterized by decreased bone mass and qualitative alterations in bone and is accompanied by an increased risk of fracture. ${ }^{1-3}$ It is a common consequence of aging that affects $\sim 200$ million individuals and causes 8.9 million fractures every year. ${ }^{4}$ In the USA and Europe, $30 \%$ of all postmenopausal women have osteoporosis. ${ }^{5}$ The diagnostic of osteoporosis by the World Health Organization (WHO) criteria is based on the measurement of bone mineral density (BMD), which is usually measured using densitometry applications such as dual X-ray photon absorptiometry. ${ }^{2,6}$ However, it is not sufficient only to do the densitometry as a diagnostic because many fragility fractures after osteoporosis occurs in the range of normal BMD values, so a clinical diagnostic should combine densitometric examination with clinical evaluations, laboratory tests, and related differential diagnoses. ${ }^{1,2}$ The National Osteoporosis Foundation suggested that treatment for osteoporosis should start with a non-pharmacologic approach, such as resistance
Correspondence: Zhenyong Zhang The Second Department of Clinical Oncology, Shengjing Hospital of China Medical University, No 39 Huaxiang Road, Tiexi District, Shenyang, Liaoning I I 0022, China

Tel +862423842568

Email zhangzy@sj-hospital.org 
and weight-bearing exercise. ${ }^{6,7}$ Bisphosphonates are typically the first-line pharmacologic agents for the treatment of patients with osteoporosis. ${ }^{89}$ However, osteonecrosis of the jaw is a known complication in a subset of patients receiving bisphosphonate treatment. ${ }^{10,11}$ Furthermore, a previous study showed that in MEPS, bisphosphonate use was increased predominantly in women (from $3.5 \%$ in 1996 to $16.6 \%$ in 2007) compared with men (2.3\% in 2007), and women experienced the vast majority of subtrochanteric fractures.

Thiazide diuretics were the first tolerated efficient antihypertensive drugs and have been used as a part of treatment strategies in numerous clinical trials involving hypertension; they are considered unequivocally effective in the treatment of hypertensive patients. ${ }^{12,13}$ Furthermore, thiazides have been regarded as candidates for the prevention of postmenopausal bone loss for many years because of their ability to decrease urinary calcium excretion at the level of the renal tubules. ${ }^{14}$ Defects in the tubular excretion of calcium are one of the main causes of osteoporosis. In a previous study, thiazide diuretics were shown to slow the rate of bone loss in older adults. ${ }^{15}$ Furthermore, it has been demonstrated that thiazides have direct effects on osteoclasts and osteoblasts. ${ }^{16,17}$ However, there is insufficient evidence that thiazides have a sustained beneficial effect in preserving bone mass and preventing osteoporosis to date.

This systematic review and meta-analysis was conducted to determine whether thiazides are beneficial for preserving bone mass and have an effect on the prevention and treatment of osteoporosis. In this study, we analyzed the effect of thiazides on serum and urinary calcium levels and BMD.

\section{Materials and methods}

\section{Search strategy}

We searched PubMed, the Cochrane Library, and Embase in June 2018 for studies using the following combination of terms: "Thiazides," "Benzothiadiazines," "Bendroflumethiazide," "Chlorothiazide," "Cyclopenthiazide," "Diazoxide," "Hydroflumethiazide," "Methyclothiazide," and "Polythiazidein" in combination with "Osteoporosis," "Osteoporoses," "Osteoporosis, Post-Traumatic," “Osteoporosis, Post Traumatic," "Post-Traumatic Osteoporoses," "Post-Traumatic Osteoporosis," "Osteoporosis, Senile," "Osteoporoses, Senile," "Senile Osteoporoses," "Senile Osteoporosis," "Osteoporosis, Involutional," "Osteoporosis, Age-Related," "Osteoporosis, Age Related," "Bone Loss, Age-Related," “Age-Related Bone Loss," "Age-Related Bone Losses," "Bone Loss, Age Related," "Bone Losses, Age-Related," “Age-Related Osteoporosis," "Age Related
Osteoporosis," "Age-Related Osteoporoses," and "Osteoporoses, Age-Related," and only English papers were included. Furthermore, the articles in reference lists of the related studies were also reviewed. Two independent reviewers examined the titles and abstracts for eligibility, and if we cannot judge whether the articles are eligible based on the titles and abstracts, the full-text article was obtained and reviewed for final inclusion. Moreover, we only included the complete original articles.

\section{Study selection}

The studies were selected according to the following inclusion criteria: 1) the studies were designed as randomized controlled trials (RCTs); 2) participants were adults with osteoporosis or the high-risk group of osteoporosis; 3 ) the thiazide intervention was compared with placebo control in the meantime patients in the control groups used placebo that did not influence bone anabolism. Furthermore, studies were excluded according to the following exclusion criteria: 1) participants had taken thiazides, unless patients had gone through a washout period; 2) the patients were allergic to thiazides formerly or currently; and 3) except for RCTs, other types of studies such as abstracts, review articles, and letters.

\section{Data extraction and quality assessment}

The following data with regard to thiazide intervention in patients with osteoporosis were extracted: 1) name of the first author, publication year; 2) participants' characteristics (gender, mean age); 3) type of intervention; 4) number of cases and participants; and 5) BMD, serum calcium, and urinary calcium from each included study. Moreover, the quality of the studies we selected was assessed according to the Cochrane risk of bias tool, including selection bias, performance bias, detection bias, attrition bias, and reporting bias. ${ }^{18}$ Each category included the following three levels: low risk, unclear risk, and high risk.

\section{Statistical analysis}

The data syntheses were performed by Review Manager Software (RevMan version 5.2; The Cochrane Collaboration, Copenhagen, Denmark). Only outcomes addressed by at least two studies were analyzed. The meta-analysis was analyzed with a random effects model. Continuous outcomes were presented as standardized mean difference (SMD) and 95\% CI. Study heterogeneity was measured using Cochran's Q statistic and $\mathrm{I}^{2}$ statistic, and it was regarded significant when $P<0.05$ for the former and $\mathrm{I}^{2}>50 \%$ for the latter. ${ }^{19,20}$ 


\section{Results}

\section{Literature search}

A total of 33 potentially relevant studies as of June 2018 were identified, 12 of which were excluded because they were duplicate publications, were not RCTs, or included exposures or end points not related to our meta-analysis. After a full-text review of the remaining studies, five publications were included. ${ }^{21-25}$ Figure 1 shows the details of the screening process.

\section{Study and patient characteristics}

The five eligible studies included 756 participants, including 614 women (81.2\%) and 142 men (18.8\%). Moreover, types of interventions included bendroflumethiazide and hydrochlorothiazide. The total number of the included patients with active treatment and placebo treatment is 372 and 384 , respectively. The duration of thiazide therapy ranged from
2 to 3 years. Overall mean age was $64.45 \pm 6.69$ (mean \pm SD) years. Table 1 shows the characteristics of the included studies in detail.

\section{Quality of trials}

The quality of the five included RCTs was assessed using the Cochrane risk of bias tool, as described in Figure 2 in detail. All the studies were judged to be at low risk of bias in terms of random sequence generation, allocation concealment, and blinding of participants and personnel. One trial was judged to be at high risk for incomplete outcome data and blinding of outcome assessment, while other trials (such as recruitment bias and contamination of the interventions) were at low risk. The rate of patients lost to follow-up was also assessed, and one trial indicated the presence of loss to follow-up, which was 3.44\%. ${ }^{22}$ Disagreement was resolved by discussion among the authors.

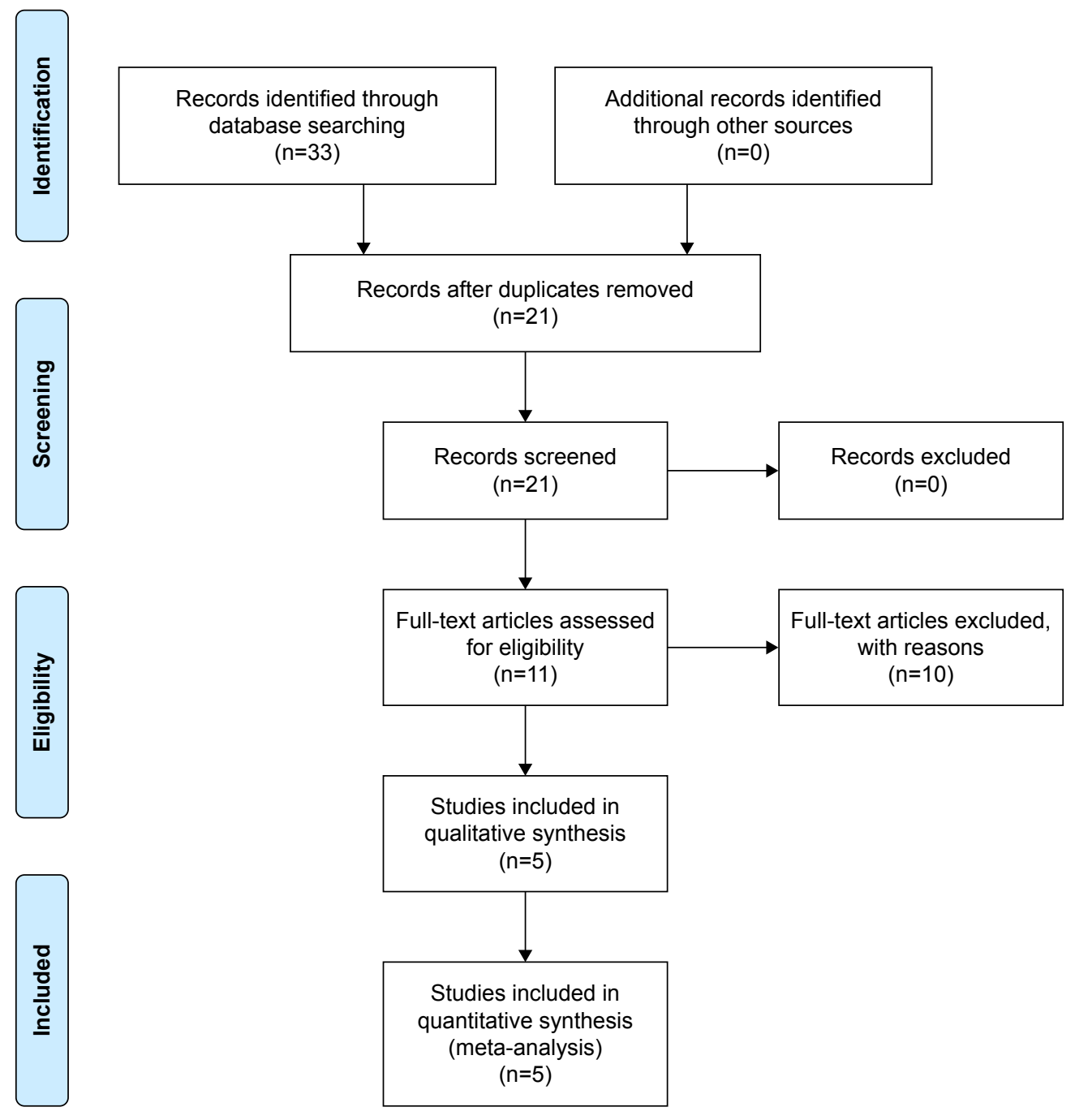

Figure I Flow diagram of the search and selection of the studies included in this meta-analysis. 


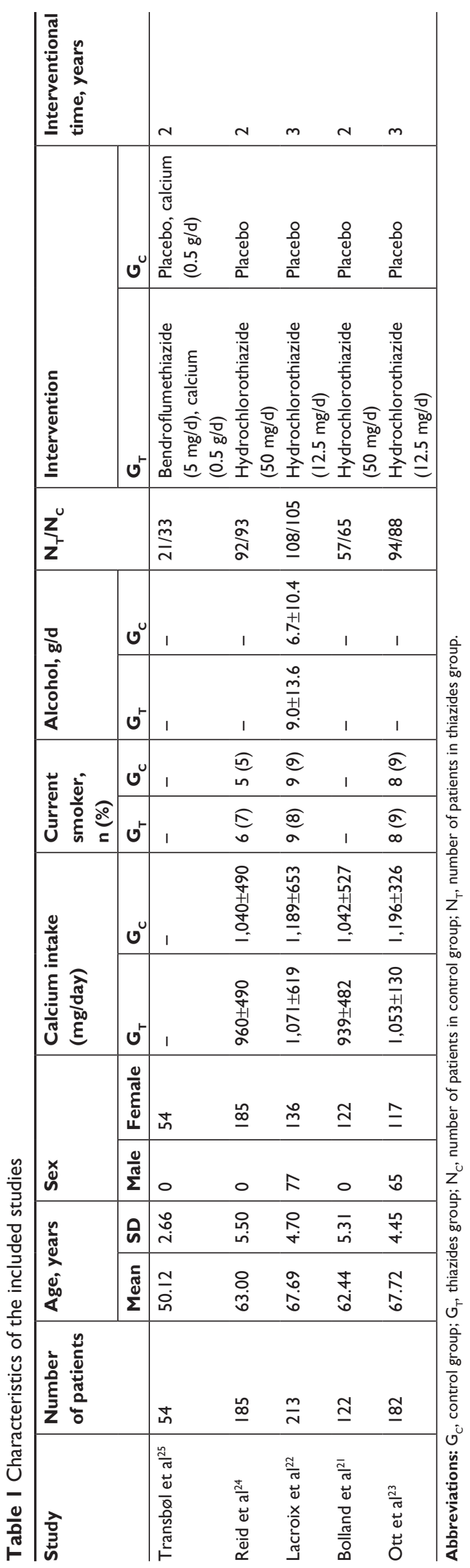

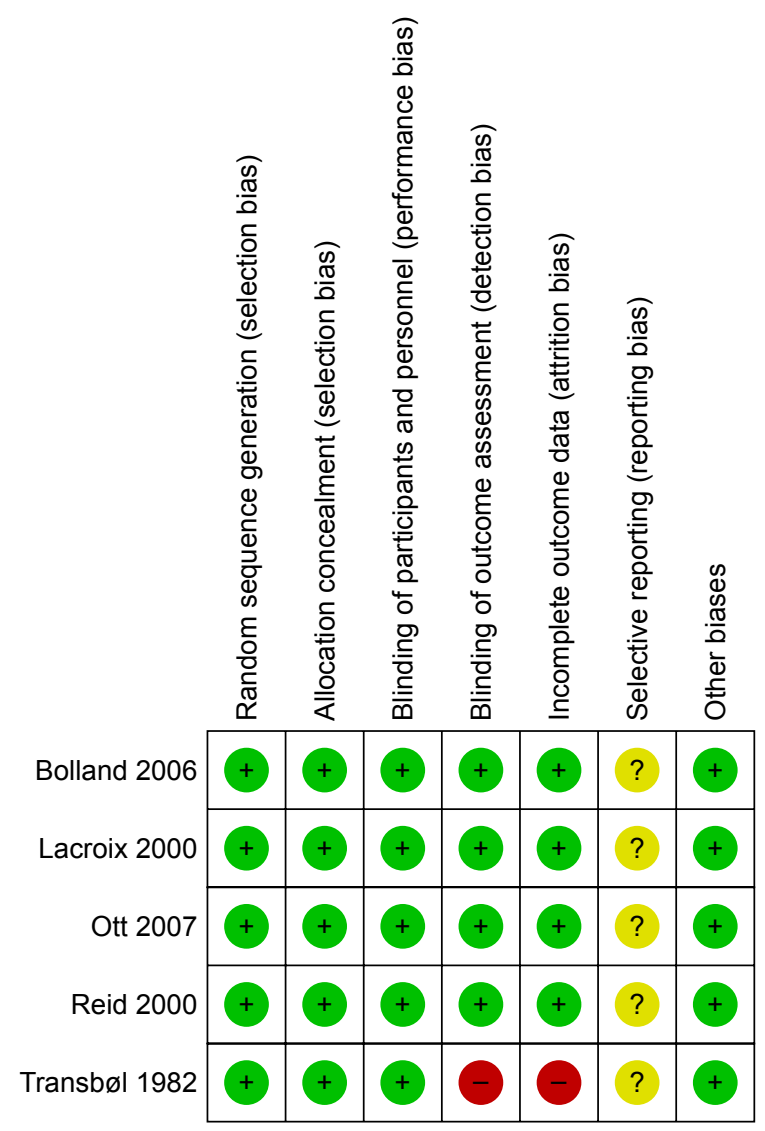

Figure 2 Risk of bias summary.

Note: Red with a minus means high risk of bias; yellow with a question mark means unclear; and green with a plus means low risk of bias.

\section{Serum calcium}

Four trials (543 patients) included an analysis of serum calcium after the intervention. The results showed that patients treated with thiazides had significantly higher calcium levels compared with patients in the control group (SMD 0.33, $95 \%$ CI $[0.16,0.50] ; \mathrm{I}^{2}$ of heterogeneity $0 \%$; random effects model) (Figure 3).

\section{Urinary calcium}

Three trials (489 patients) included an analysis of urinary calcium after the intervention. The results showed that patients in the thiazide group showed significantly lower urinary calcium levels compared with the control groups (SMD $-0.35,95 \%$ CI $[-0.52,-0.17] ; \mathrm{I}^{2}$ of heterogeneity $0 \%$; random effects model) (Figure 4).

\section{BMD}

BMD data obtained using dual-energy X-ray absorptiometry were analyzed in two studies involving 398 patients (200 in the thiazide group and 198 in the control group). ${ }^{22,24}$ The results showed that patients treated with thiazides had no 


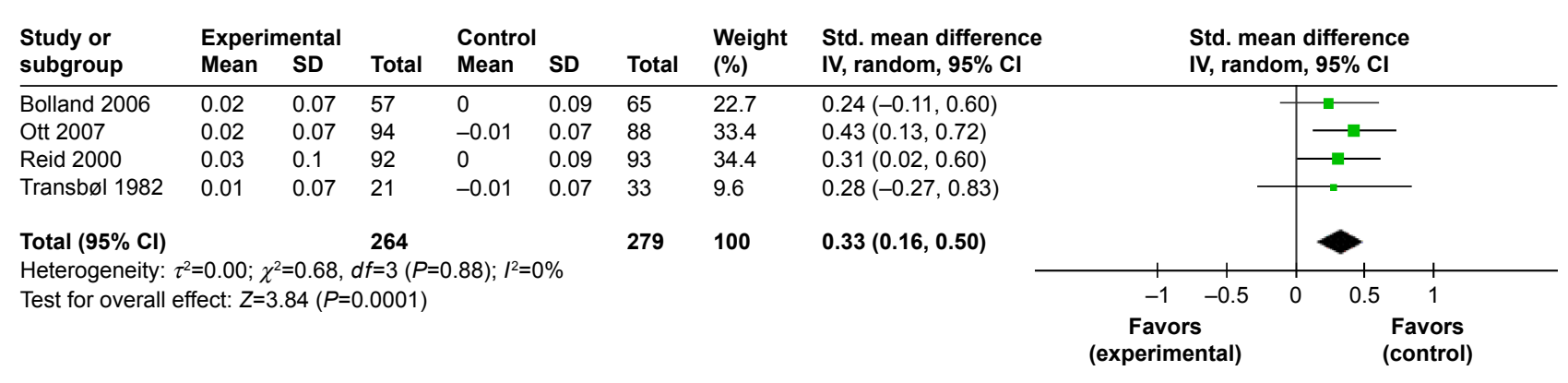

Figure 3 Forest plot for serum calcium.

Note: A comparison of thiazide treatment with placebo treatment on serum calcium in patients with osteoporosis.

statistically significant difference in BMD compared with the control group (SMD 0.19, 95\% CI [-0.16, 0.54]; I I of heterogeneity 68\%; random effects model) (Figure 5). Although significant heterogeneity was evident, sensitivity analysis was not performed due to the small number of trials. The significant heterogeneity could be attributed to the different methods of evaluation used in the trials.

\section{Discussion}

Thiazides have been widely used as antihypertensive drugs with a tolerable efficiency. ${ }^{26}$ Previous studies have shown that thiazides may also affect bone metabolism and BMD. ${ }^{27,28}$ However, the effectiveness of thiazides in the treatment of osteoporosis has not been fully elucidated. The present metaanalysis summarized the results of the five included RCTs that assessed the effects of thiazides on the BMD and mineral metabolism in the elderly. The findings suggest that thiazides have some benefits for preserving bone mass and may be effective in the prevention and treatment of osteoporosis.

BMD in the thiazide group did not differ significantly from that in the control group. The quality of this evidence was assessed to be very low because only two RCTs provided BMD data. ${ }^{22,24}$ Therefore, insufficient data limited the quality of evidence. Four of the RCTs indicated that thiazides had a positive effect on BMD. ${ }^{21-24}$ Thus, there is evidence to support the beneficial effects of thiazides on BMD, but this was not confirmed in this analysis. If we want to confirm that thiazides have beneficial effect on BMD, more high-quality RCTs are needed in the future.

Measurement of serum calcium should be one of the firstline investigations in patients with osteoporosis. ${ }^{1}$ Elevated serum calcium is associated with a wide range of adverse health outcomes including osteoporosis, and decreased urinary calcium excretion can lead to increased serum calcium levels. In the current meta-analysis, the results suggest that the use of thiazides could lead to a significant increase in serum calcium level compared with that in the control group. Furthermore, urinary calcium excretion has been shown to be low in many postmenopausal women and in the elderly because of impaired intestinal calcium absorption. ${ }^{29,30}$ Previous studies have reported that thiazides can reduce urinary calcium excretion by $25 \%-40 \%$, potentially improving bone mass and reducing fracture risk, while intestinal calcium absorption keeps on persistently elevated. ${ }^{31-35}$ In these results, urinary calcium excretion declined significantly in the thiazide group. There was no heterogeneity in these results, which could reach statistical significance in the current meta-analysis. To sum up, combining the results, the meta-analysis indicated that thiazides have the possible effect on the prevention and treatment of osteoporosis.

Certain limitations of this meta-analysis should be acknowledged. First, evidence of the benefit was limited

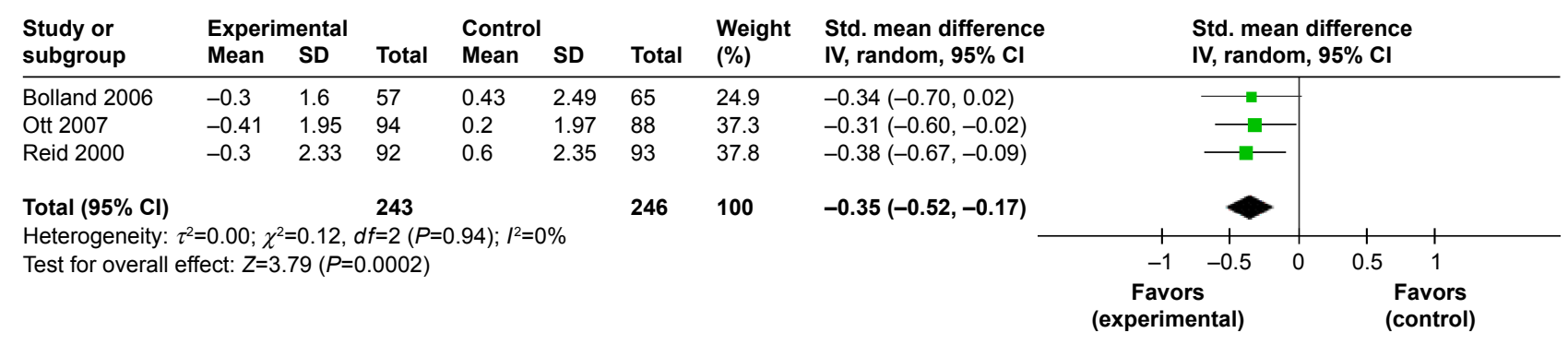

Figure 4 Forest plot for urinary calcium.

Note: A comparison of thiazide treatment with placebo treatment on urinary calcium in patients with osteoporosis. 


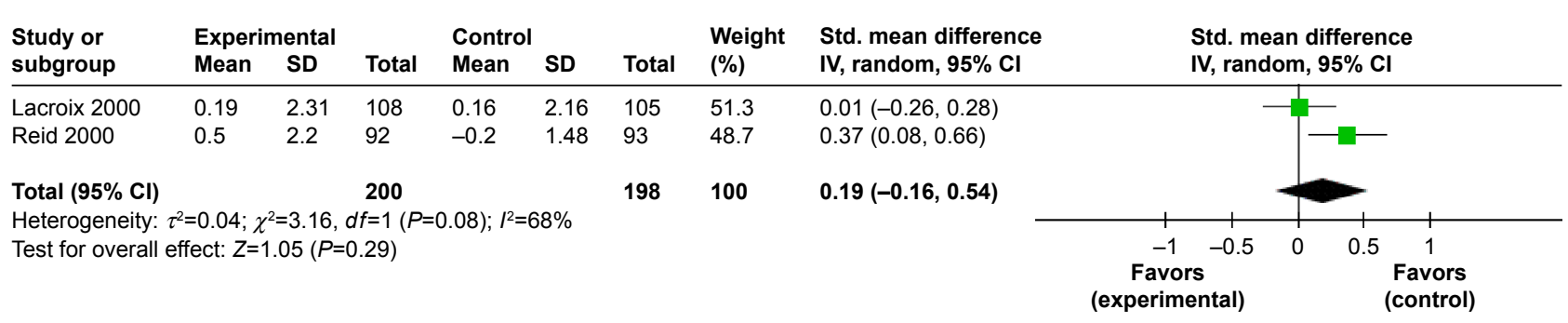

Figure 5 Forest plot for bone mineral density.

Note: A comparison of thiazide treatment with placebo treatment on bone mineral density in patients with osteoporosis.

because of the small number of RCTs. Second, not all the included studies provided all the data that we sought; for example, although two studies reported significant between-group differences in BMD, the data provided were not detailed. Thus, quality of the evidence was limited by insufficient data. Third, this meta-analysis included the only papers published in English language, and so trials published in other languages were systematically excluded. Fourth, over $81.2 \%(\mathrm{n}=614)$ of the included subjects were females and only $18.8 \%(\mathrm{n}=142)$ of the included subjects were males, so the results of this study are more applicable to females. Despite these limitations, this study supports the use of thiazides to increase serum calcium levels and decrease urinary calcium excretion in patients with osteoporosis.

\section{Conclusion}

The results of this meta-analysis demonstrated that thiazides might play a role in preserving bone mass in patients with osteoporosis and have the possible effect on the prevention and treatment of osteoporosis. However, we must interpret the results cautiously because of some limitations, and future high-quality trials are needed to confirm these findings.

\section{Disclosure}

The authors report no conflicts of interest in this work.

\section{References}

1. Rossini M, Adami S, Bertoldo F, et al. Guidelines for the diagnosis, prevention and management of osteoporosis. Reumatismo. 2016;68(1):1-39.

2. Ulivieri FM, Caudarella R, Camisasca M. Assessment of bone quality in osteoporosis treatment with bone anabolic agents: really something new? Curr Rheumatol Rev. 2018;14(1):53-61.

3. Shi Z, Zhou H, Pan B, et al. Effectiveness of Teriparatide on Fracture Healing: A Systematic Review and Meta-Analysis. PLoS One. 2016; 11(12):e0168691.

4. Hadji P, Jacob L, Kostev K. Gender- and age-related treatment compliance in patients with osteoporosis in Germany. Patient Prefer Adherence. 2016;10:2379-2385.

5. Bock O, Felsenberg D. Bisphosphonates in the management of postmenopausal osteoporosis - optimizing efficacy in clinical practice. Clin Interv Aging. 2008;3(2):279-297.
6. Cosman F, de Beur SJ, Leboff MS, et al. Clinician's Guide to Prevention and Treatment of Osteoporosis. Osteoporos Int. 2014;25(10): 2359-2381.

7. Hinton PS, Nigh P, Thyfault J. Effectiveness of resistance training or jumping-exercise to increase bone mineral density in men with low bone mass: A 12-month randomized, clinical trial. Bone. 2015;79: 203-212.

8. Black DM, Bauer DC, Schwartz AV, Cummings SR, Rosen CJ. Continuing bisphosphonate treatment for osteoporosis - for whom and for how long? N Engl J Med. 2012;366(22):2051-2053.

9. Rodan G, Reszka A, Golub E, Rizzoli R. Bone safety of long-term bisphosphonate treatment. Curr Med Res Opin. 2004;20(8):1291-1300.

10. Oteri G, Bramanti E, Nigrone V, Cicciù M. Decayed, Missing, and Filled Teeth Index and Periodontal Health in Osteoporotic Patients Affected by BRONJ: An Observational Study. J Osteoporos. 2013;2013:231289.

11. Nastro Siniscalchi E, Allegra A, De Ponte FS, et al. Spontaneous Healing of Clodronate-Related Osteonecrosis of the Jaw. J Craniofac Surg. 2017;28(7):e687-e689.

12. Reilly RF, Peixoto AJ, Desir GV. The evidence-based use of thiazide diuretics in hypertension and nephrolithiasis. Clin J Am Soc Nephrol. 2010;5(10):1893-1903.

13. Salvetti A, Ghiadoni L. Thiazide diuretics in the treatment of hypertension: an update. J Am Soc Nephrol. 2006;17(4 Suppl 2):S25-S29.

14. Heshmati HM, Khosla S, Burritt MF, O'Fallon WM, Riggs BL. A defect in renal calcium conservation may contribute to the pathogenesis of postmenopausal osteoporosis. J Clin Endocrinol Metab. 1998; 83(6):1916-1920

15. Wasnich R, Davis J, Ross P, Vogel J. Effect of thiazide on rates of bone mineral loss: a longitudinal study. BMJ. 1990;301(6764):1303-1305.

16. Hall TJ, Schaueblin M. Hydrochlorothiazide inhibits osteoclastic bone resorption in vitro. Calcif Tissue Int. 1994;55(4):266-268.

17. Lajeunesse D, Delalandre A, Guggino SE. Thiazide diuretics affect osteocalcin production in human osteoblasts at the transcription level without affecting vitamin D3 receptors. J Bone Miner Res. 2000;15(5): 894-901.

18. Higgins JP, Altman DG, Gotzsche PC, et al; Cochrane Bias Methods Group; Cochrane Statistical Methods Group. The Cochrane Collaboration's tool for assessing risk of bias in randomised trials. BMJ. 2011;343:d5928.

19. Higgins JP, Thompson SG. Quantifying heterogeneity in a metaanalysis. Stat Med. 2002;21(11):1539-1558.

20. Shi Z, Zhou H, Lu L, et al. Aquatic Exercises in the Treatment of Low Back Pain: A Systematic Review of the Literature and Meta-Analysis of Eight Studies. Am J Phys Med Rehabil. 2018;97(2):116-122.

21. Bolland MJ, Ames RW, Horne AM, Orr-Walker BJ, Gamble GD, Reid IR. The effect of treatment with a thiazide diuretic for 4 years on bone density in normal postmenopausal women. Osteoporos Int. 2007; 18(4):479-486.

22. Lacroix AZ, Ott SM, Ichikawa L, Scholes D, Barlow WE. Low-dose hydrochlorothiazide and preservation of bone mineral density in older adults. A randomized, double-blind, placebo-controlled trial. Ann Intern Med. 2000;133(7):516-526. 
23. Ott SM, LaCroix AZ, Scholes D, Ichikawa LE, Wu K. Effects of three years of low-dose thiazides on mineral metabolism in healthy elderly persons. Osteoporos Int. 2008;19(9):1315-1322.

24. Reid IR, Ames RW, Orr-Walker BJ, et al. Hydrochlorothiazide reduces loss of cortical bone in normal postmenopausal women: a randomized controlled trial. Am J Med. 2000;109(5):362-370.

25. Transbøl I, Christensen MS, Jensen GF, Christiansen C, McNair P. Thiazide for the postponement of postmenopausal bone loss. Metabolism. 1982;31(4):383-386.

26. Sinha AD, Agarwal R. Thiazides are useful agents in CKD. J Am Soc Hypertens. 2016;10(4):288-289.

27. Morton DJ, Barrett-Connor EL, Edelstein SL. Thiazides and bone mineral density in elderly men and women. Am J Epidemiol. 1994; 139(11):1107-1115.

28. Rejnmark L, Vestergaard P, Pedersen AR, Heickendorff L, Andreasen F, Mosekilde L. Dose-effect relations of loop- and thiazide-diuretics on calcium homeostasis: a randomized, double-blinded Latin-square multiple cross-over study in postmenopausal osteopenic women. Eur J Clin Invest. 2003;33(1):41-50.

29. Gallagher JC, Riggs BL, Eisman J, Hamstra A, Arnaud SB, DeLuca HF. Intestinal calcium absorption and serum vitamin $\mathrm{D}$ metabolites in normal subjects and osteoporotic patients: effect of age and dietary calcium. J Clin Invest. 1979;64(3):729-736
30. Sakhaee K, Nicar MJ, Glass K, Zerwekh JE, Pak CY. Reduction in intestinal calcium absorption by hydrochlorothiazide in postmenopausal osteoporosis. J Clin Endocrinol Metab. 1984;59(6):1037-1043.

31. Pak CY, Nicar M, Northcutt C. The definition of the mechanism of hypercalciuria is necessary for the treatment of recurrent stone formers. Contrib Nephrol. 1982;33:136-151.

32. Adams JS, Song CF, Kantorovich V. Rapid recovery of bone mass in hypercalciuric, osteoporotic men treated with hydrochlorothiazide. Ann Intern Med. 1999;130(8):658-660.

33. Feskanich D, Willett WC, Stampfer MJ, Colditz GA. A prospective study of thiazide use and fractures in women. Osteoporos Int. 1997; 7(1):79-84.

34. Heaney RP. Lifelong calcium intake and prevention of bone fragility in the aged. Calcif Tissue Int. 1991;49 Suppl(S1):S42-S45.

35. Lamberg BA, Kuhlback B. Effect of chlorothiazide and hydrochlorothiazide on the excretion of calcium in urine. Scand J Clin Lab Invest. 1959;11(4):351-357.

\section{Publish your work in this journal}

Drug Design, Development and Therapy is an international, peerreviewed open-access journal that spans the spectrum of drug design and development through to clinical applications. Clinical outcomes, patient safety, and programs for the development and effective, safe, and sustained use of medicines are the features of the journal, which has also been accepted for indexing on PubMed Central. The manuscript management system is completely online and includes a very quick and fair peer-review system, which is all easy to use. Visit http://www.dovepress.com/testimonials.php to read real quotes from published authors.

Submit your manuscript here: http://www.dovepress.com/drug-design-development-and-therapy-journal 